\title{
Counting segmented permutations using bicoloured Dyck paths
}

\author{
Anders Claesson \\ Division of Mathematics, \\ Department of Chemistry and Biomedical Sciences, \\ University of Kalmar, Sweden \\ anders.claesson@hik.se
}

Submitted: Jun 14, 2005; Accepted: Aug 9, 2005; Published: Aug 17, 2005

Mathematics Subject Classifications: 05A05, 05A15

\begin{abstract}
A bicoloured Dyck path is a Dyck path in which each up-step is assigned one of two colours, say, red and green. We say that a permutation $\pi$ is $\sigma$-segmented if every occurrence $o$ of $\sigma$ in $\pi$ is a segment-occurrence (i.e., $o$ is a contiguous subword in $\pi$ ).

We show combinatorially the following results: The 132-segmented permutations of length $n$ with $k$ occurrences of 132 are in one-to-one correspondence with bicoloured Dyck paths of length $2 n-4 k$ with $k$ red up-steps. Similarly, the 123segmented permutations of length $n$ with $k$ occurrences of 123 are in one-to-one correspondence with bicoloured Dyck paths of length $2 n-4 k$ with $k$ red up-steps, each of height less than 2 .

We enumerate the permutations above by enumerating the corresponding bicoloured Dyck paths. More generally, we present a bivariate generating function for the number of bicoloured Dyck paths of length $2 n$ with $k$ red up-steps, each of height less than $h$. This generating function is expressed in terms of Chebyshev polynomials of the second kind.
\end{abstract}

\section{Introduction}

Let $\mathcal{S}_{n}$ be the set of permutation of $[n]=\{1,2, \ldots, n\}$. Let $\pi \in \mathcal{S}_{n}$ and $\sigma \in \mathcal{S}_{k}$, with $k \leq n$. An occurrence of $\sigma$ in $\pi$ is a subword $o$ of length $k$ in $\pi$ such that $o$ and $\sigma$ are in same relative order. In this context $\sigma$ is called a pattern. For example, an occurrence of the pattern 132 in $\pi$ is a subword $\pi(i) \pi(j) \pi(k)$ such that $\pi(i)<\pi(k)<\pi(j)$; so 253 is an occurrence of 132 in 42513. A permutation $\pi$ that does not contain any occurrence of $\sigma$ is said to avoid $\sigma$. 
It is relatively easy to show that number of permutations of $[n]$ avoiding a pattern of length 3 is the Catalan number, $C_{n}=\left(\begin{array}{c}2 n \\ n\end{array}\right) /(n+1)$ (e.g., see [9] or [5]). In contrast, to count the permutations containing $r$ occurrences of a fixed pattern of length 3 , for a general $r$, is a very hard problem. The best result on this latter problem has been achieved by Mansour and Vainshtein [7]. They presented an algorithm that computes the generating function for the number of permutations with $r$ occurrences of 132 for any $r \geq 0$. The algorithm has been implemented in C. It yields explicit results for $1 \leq r \leq 6$.

We say that an occurrence $o$ of $\sigma$ in $\pi$ is a segment-occurrence if $o$ is a segment (also called factor) of $\pi$, in other words, if $o$ is a contiguous subword in $\pi$. Elizalde and Noy [2] presented exponential generating functions for the distribution of the number of segmentoccurrences of any pattern of length 3 . Related problems have also been studied by Kitaev [3] and by Kitaev and Mansour [4].

We say that $\pi$ is $\sigma$-segmented if every occurrence of $\sigma$ in $\pi$ is a segment-occurrence. For instance, 4365172 contains 3 occurrences of 132, namely 465, 365, and 172. Of these occurrences, only 365 and 172 are segment-occurrences. Thus 4365172 is not 132 -segmented. Note that if $\pi$ is $\sigma$-avoiding then $\pi$ is also $\sigma$-segmented. In this article we try to enumerate the $\sigma$-segmented permutations by length and by the number of occurrences of $\sigma$.

Krattenthaler [5] gave two bijections: one between 132-avoiding permutations and Dyck paths, and one between 123-avoiding permutations and Dyck paths. We obtain two new results by extending these bijections:

- The 132-segmented permutations of length $n$ with $k$ occurrences of 132 are in one-toone correspondence with bicoloured Dyck paths of length $2 n-4 k$ with $k$ red up-steps.

- The 123-segmented permutations of length $n$ with $k$ occurrences of 123 are in one-toone correspondence with bicoloured Dyck paths of length $2 n-4 k$ with $k$ red up-steps, each of height less than 2 .

Here a bicoloured Dyck path is a Dyck path in which each up-step is assigned one of two colours, say, red and green. We enumerate the permutations above by enumerating the corresponding bicoloured Dyck paths. To be more precise, let $\mathcal{B}_{n, k}$ be the set of bicoloured Dyck path of length $2 n$ with $k$ red up-steps. Let $\mathcal{B}_{n, k}^{[h]}$ be the subset of $\mathcal{B}_{n, k}$ consisting of those paths where the height of each red up-step is less than $h$. It is plain that $\left|\mathcal{B}_{n, k}\right|=\left(\begin{array}{l}n \\ k\end{array}\right) C_{n}$. We show that

$$
\sum_{n, k \geq 0}\left|\mathcal{B}_{n, k}^{[h]}\right| q^{k} t^{n}=\frac{C(t)-2 x q U_{h}(x) U_{h-1}(x)}{1+q-q U_{h}^{2}(x)}, \quad x=\frac{1}{2 \sqrt{(1+q) t}}
$$

where $C(t)=(1-\sqrt{1-4 t}) /(2 t)$ is the generating function for the Catalan numbers, and $U_{n}(x)$ is the $n$th Chebyshev polynomial of the second kind. We also find formulas for $\left|\mathcal{B}_{n, k}^{[1]}\right|$ and $\left|\mathcal{B}_{n, k}^{[2]}\right|$. 


\section{Bicoloured Dyck paths}

By a lattice path we shall mean a path in $\mathbb{Z}^{2}$ with steps $(1,1)$ and $(1,-1)$; the steps $(1,1)$ and $(1,-1)$ will be called up- and down-steps, respectively. Furthermore, a lattice path that never falls below the $x$-axis will be called nonnegative.A Dyck path of length $2 n$ is a nonnegative lattice path from $(0,0)$ to $(2 n, 0)$. As an example, these are the 5 Dyck paths of length 6 :

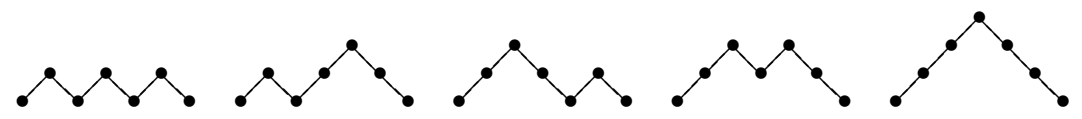

Letting $u$ and $d$ represent the steps $(1,1)$ and $(1,-1)$, we code a Dyck path with a word over $\{u, d\}$. For example, the paths above are coded by

\section{ududud uduudd uuddud uududd uuuddd}

Let $\mathcal{D}_{n}$ be the language over $\{u, d\}$ obtained from Dyck paths of length $2 n$ via this coding, and let $\mathcal{D}=\cup_{n \geq 0} \mathcal{D}_{n}$. In general, if $\mathcal{A}$ is a language over some alphabet $X$, then the characteristic series of $\mathcal{A}$, also (by slight abuse of notation) denoted $\mathcal{A}$, is the element of $\mathbb{C}\langle\langle X\rangle\rangle$ defined by

$$
\mathcal{A}=\sum_{w \in \mathcal{A}} w
$$

A nonempty Dyck path $\beta$ can be written uniquely as $u \beta_{1} d \beta_{2}$ where $\beta_{1}$ and $\beta_{2}$ are Dyck paths. This decomposition is called the first return decomposition of $\beta$, because the $d$ in $u \beta_{1} d \beta_{2}$ corresponds to the first place, after $(0,0)$, where the path touches the $x$-axis. By this decomposition, the characteristic series of $\mathcal{D}$ is uniquely determined by the functional equation

$$
\mathcal{D}=\epsilon+u \mathcal{D} d \mathcal{D}
$$

where $\epsilon$ denotes the empty word.

In a similar vein, we now consider the language $\mathcal{B}$ over $\{u, \bar{u}, d\}$ whose characteristic series is uniquely determined by the functional equation

$$
\mathcal{B}=\epsilon+(u+\bar{u}) \mathcal{B} d \mathcal{B} \text {. }
$$

Let $\mathcal{B}_{n}$ be the set of words in $\mathcal{B}$ that are of length $2 n$, and let $\mathcal{B}_{n, k}$ be the set of words in $\mathcal{B}_{n}$ with $k$ occurrences of $\bar{u}$. As an example, when $n=3$ and $k=1$ there are 15 such words, namely

$\begin{array}{lllll}\bar{u} d u d u d & \bar{u} d u u d d & \bar{u} u d d u d & \bar{u} u d u d d & \bar{u} u u d d d \\ u d \bar{u} d u d & u d \bar{u} u d d & u \bar{u} d d u d & u \bar{u} d u d d & u \bar{u} u d d d \\ u d u d \bar{u} d & u d u \bar{u} d d & u u d d \bar{u} d & u u d \bar{u} d d & u u \bar{u} d d d\end{array}$

We may view the elements of $\mathcal{B}$ as bicoloured Dyck paths. The words from the previous example are depicted below. 


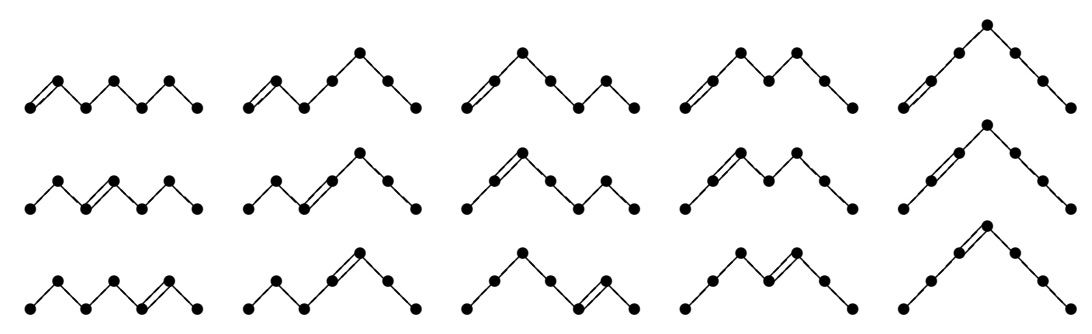

Here steps represented by double edges are, say, red, and steps represented by simple edges are, say, green.

Proposition 1 With $C_{n}=\left|\mathcal{D}_{n}\right|$, we have

$$
\left|\mathcal{B}_{n, k}\right|=\left(\begin{array}{l}
n \\
k
\end{array}\right) C_{n} \quad \text { and } \quad\left|\mathcal{B}_{n}\right|=2^{n} C_{n} .
$$

Proof A bicoloured Dyck paths $\beta$ of length $2 n$ naturally breaks up into two parts: (a) The Dyck path obtained from $\beta$ by removing colours. (b) The subset of $[n]$ consisting of those integers $i$ for which the $i$ th up-step is red.

For $h \geq 1$, let $\mathcal{B}^{[h]}$ be the subset of $\mathcal{B}$ whose characteristic series is the solution to

$$
\mathcal{B}^{[h]}=\epsilon+(u+\bar{u}) \mathcal{B}^{[h-1]} d \mathcal{B}^{[h]}
$$

with the initial condition $\mathcal{B}^{[0]}=\mathcal{D}$, where $\mathcal{D}$ is defined as above. Let

$$
\begin{aligned}
& \mathcal{B}_{n}^{[h]} \text { be the set of words in } \mathcal{B}^{[h]} \text { that are of length } 2 n \text {, and let } \\
& \mathcal{B}_{n, k}^{[h]} \text { be the set of words in } \mathcal{B}_{n}^{[h]} \text { with } k \text { occurrences of } \bar{u} \text {. }
\end{aligned}
$$

To translate these definitions in terms of lattice paths we define the height of a step in a (bicoloured) lattice path as the height above the $x$-axis of its left point. Then $\mathcal{B}^{[h]}$ is the set of bicoloured Dyck paths whose red up-steps all are of height less than $h$. As an example, there is exactly one element in $\mathcal{B}_{3,1}$ that is not in $\mathcal{B}^{[2]}$, namely

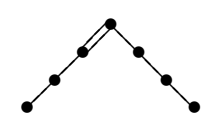

To count words of given length in $\mathcal{D}, \mathcal{B}$ and $\mathcal{B}^{[h]}$, we will study the commutative counterparts of the functional equations (1), (2) and (3). Formally, we define the substitution $\mu: \mathbb{C}\langle\langle u, \bar{u}, d\rangle\rangle \rightarrow \mathbb{C}[[q, t]]$ by

$$
\mu=\{u \mapsto 1, \bar{u} \mapsto q, d \mapsto t\}
$$

Let $C=\mu(\mathcal{D}), B=\mu(\mathcal{B})$, and $B^{[h]}=\mu\left(\mathcal{B}^{[h]}\right)$. We then get

$$
\begin{aligned}
C & =1+t C^{2}, \\
B & =1+(1+q) t B^{2}, \\
B^{[h]} & =1+(1+q) t B^{[h-1]} B^{[h]}, \quad B^{[0]}=C .
\end{aligned}
$$


By an easy application of the Lagrange inversion formula it follows from (4) that

$$
\left[t^{n}\right](C(t))^{i}=\frac{i}{i+n}\left(\begin{array}{c}
2 n+i-1 \\
n
\end{array}\right)
$$

In particular, we obtain that $C(t)$ is the familiar generating function of the Catalan numbers, $C_{n}=\frac{1}{n+1}\left(\begin{array}{c}2 n \\ n\end{array}\right)$. Thus we have derived the well known fact that the number of Dyck paths of length $2 n$ is the $n$th Catalan number. Furthermore, it follows from (5) that

$$
B(q, t)=C((1+q) t)
$$

and it follows from (6) that

$$
B^{[h]}(q, t)=\frac{1}{1-(1+q) t B^{[h-1]}}, \quad B^{[0]}=C .
$$

From these series we generate the first few values of $\left|\mathcal{B}_{n, k}\right|,\left|\mathcal{B}_{n, k}^{[1]}\right|$ and $\left|\mathcal{B}_{n, k}^{[2]}\right| ;$ tables with these values are given in Section 5 .

Recall that the Chebyshev polynomials of the second kind, denoted $U_{n}(x)$, are defined by

$$
U_{n}(x)=\frac{\sin (n+1) \theta}{\sin \theta}
$$

where $n$ is an integer, $x=\cos \theta$, and $0 \leq \theta \leq \pi$. Equivalently, these polynomials can be defined as the solution to the linear difference equation

$$
U_{n+1}(x)=2 x U_{n}(x)-U_{n-1}(x),
$$

with $U_{-1}(x)=0$ and $U_{0}(x)=1$.

In 1970 Kreweras [6] showed that

$$
C^{[h]}(t)=\frac{U_{h}\left(\frac{1}{2 \sqrt{t}}\right)}{\sqrt{t} \cdot U_{h+1}\left(\frac{1}{2 \sqrt{t}}\right)}
$$

is the generating function for Dyck paths that stay below height $h$. Note that, since $C^{[0]}=1$ and $C^{[h]}=\left(1-t C^{[h-1]}\right)^{-1}$, this result is also easy to prove by induction on $h$.

Theorem 2 With $B^{[h]}$ being the generating function for the number of Dyck paths whose red up-steps all are of height less than $h$, and $U_{n}$ being the nth Chebyshev polynomial of the second kind we have

$$
B^{[h]}(q, t)=\frac{4 x^{2} U_{h-1}(x)-2 x U_{h-2}(x) C(t)}{2 x U_{h}(x)-U_{h-1}(x) C(t)}=\frac{C(t)-2 x q U_{h}(x) U_{h-1}(x)}{1+q-q U_{h}^{2}(x)},
$$

where $x=1 /(2 \sqrt{(1+q) t})$, and $C(t)=(1-\sqrt{1-4 t}) /(2 t)$ is the generating function for the Catalan numbers. 
Proof We shall prove the first equality by induction. To this end, we let

$$
F^{[h]}(q, t)=\frac{4 x^{2} U_{h-1}(x)-2 x U_{h-2}(x) C(t)}{2 x U_{h}(x)-U_{h-1}(x) C(t)} .
$$

From $U_{-2}(x)=-1, U_{-1}(x)=0$, and $U_{0}(x)=1$ it readily follows that $F^{[0]}(q, t)=C(t)=$ $B^{[0]}(q, t)$. If $B^{[h]}=F^{[h]}$, for some fixed $h \geq 0$, then

$$
\begin{aligned}
B^{[h+1]} & =\frac{1}{1-(1+q) t B^{[h]}} \\
& =\frac{1}{1-(1+q) t F^{[h]}} \\
& =\frac{2 x U_{h}-U_{h-1} C}{2 x U_{h}-U_{h-1} C-(1+q) t\left(4 x^{2} U_{h-1}-2 x U_{h-2} C\right)} \\
& =\frac{2 x U_{h}-U_{h-1} C}{2 x U_{h}-(1+q) t 4 x^{2} U_{h-1}-\left(U_{h-1}-(1+q) t 2 x U_{h-2}\right) C} \\
& =\frac{4 x^{2} U_{h}-2 x U_{h-1} C}{2 x\left(2 x U_{h}-(1+q) t 4 x^{2} U_{h-1}\right)-\left(2 x U_{h-1}-(1+q) t 4 x^{2} U_{h-2}\right) C} \\
& =\frac{4 x^{2} U_{h}-2 x U_{h-1} C}{2 x\left(2 x U_{h}-U_{h-1}\right)-\left(2 x U_{h-1}-U_{h-2}\right) C} \\
& =\frac{4 x^{2} U_{h}-2 x U_{h-1} C}{2 x U_{h+1}-U_{h} C} \\
& =F^{[h+1]},
\end{aligned}
$$

in which $U_{h}=U_{h}(x)$ and $C=C(t)$. This completes the induction step, and thus the first equality holds for all $h \geq 0$. The second equality is plain algebra/trigonometry.

Proposition 3 For $n, k \geq 0$ we have

$$
\begin{aligned}
\left|\mathcal{B}_{n, k}^{[1]}\right| & =b(n+k, n-k)=\frac{2 k+1}{n+k+1}\left(\begin{array}{c}
2 n \\
n-k
\end{array}\right), \\
\left|\mathcal{B}_{n}^{[1]}\right| & =\left(\begin{array}{c}
2 n \\
n
\end{array}\right)
\end{aligned}
$$

where $b(n, k)=\frac{n-k+1}{n+1}\left(\begin{array}{c}n+k \\ n\end{array}\right)$ is a ballot number.

Proof The ballot number $b(n, k)$ is the number of nonnegative lattice paths from $(0,0)$ to $(n+k, n-k)$. Thus, the first claim of the proposition is that $\left|\mathcal{B}_{n, k}^{[1]}\right|$ equals the number of nonnegative lattice paths from $(0,0)$ to $(2 n, 2 k)$. Let $\mathcal{A}_{n, k}$ denote the language over $\{u, d\}$ obtained from these paths via the usual coding. In addition, let $\mathcal{A}_{n}=\cup_{k \geq 0} \mathcal{A}_{n, k}$ and $\mathcal{A}=\cup_{n \geq 0} \mathcal{A}_{n}$. The characteristic series of $\mathcal{A}$ satisfies

$$
\mathcal{A}=\epsilon+u \mathcal{D}(u+d) \mathcal{A} \text {. }
$$


From (3) we also know that

$$
\mathcal{B}^{[1]}=\epsilon+(u+\bar{u}) \mathcal{D} d \mathcal{B}^{[1]}
$$

We exploit the obvious similarity between these two functional equations to define, by recursion, a length preserving bijection $f$ from $\mathcal{B}^{[1]}$ onto $\mathcal{A}$ such that $\beta \in \mathcal{B}^{[1]}$ has exactly $k$ occurrences of $\bar{u}$ precisely when $f(\beta) \in \mathcal{A}$ ends at height $2 k$ :

$$
f(\beta)= \begin{cases}\epsilon & \text { if } \beta=\epsilon \\ u \beta_{1} d f\left(\beta_{2}\right) & \text { if } \beta=u \beta_{1} d \beta_{2}, \beta_{1} \in \mathcal{D}, \beta_{2} \in \mathcal{B}^{[1]} \\ u \beta_{1} u f\left(\beta_{2}\right) & \text { if } \beta=\bar{u} \beta_{1} d \beta_{2}, \beta_{1} \in \mathcal{D}, \beta_{2} \in \mathcal{B}^{[1]}\end{cases}
$$

For $\beta \in \mathcal{B}^{[1]}$, let $|\beta|_{\bar{u}}$ denote the number of occurrences of $\bar{u}$ in $\beta$, and for $\alpha \in \mathcal{A}$ let $h(\alpha)$ denote the height at which $\alpha$ ends. To prove that $f$ is length preserving, bijective, and that $2|\cdot|_{\bar{u}}=h \circ f$, we use induction on path-length: $f$ trivially has these properties as a function from $\mathcal{B}_{0}^{[1]}$ to $\mathcal{A}_{0}$. Let $n$ be a positive integer and assume that $f$ has the desired properties as a function from $\cup_{k=0}^{n-1} \mathcal{B}_{k}^{[1]}$ to $\cup_{k=0}^{n-1} \mathcal{A}_{k}$. Any $\beta$ in $\mathcal{B}_{n}^{[1]}$ can be written as $\beta=x \beta_{1} d \beta_{2}$ for some $x \in\{u, \bar{u}\}, \beta_{1} \in \mathcal{D}$ and $\beta_{2} \in \mathcal{B}^{[1]}$. Therefore, using induction,

$$
|f(\beta)|=2+\left|\beta_{1}\right|+\left|f\left(\beta_{2}\right)\right|=2+\left|\beta_{1}\right|+\left|\beta_{2}\right|=|\beta|
$$

and

$$
(h \circ f)(\beta)=2|x|_{\bar{u}}+(h \circ f)\left(\beta_{2}\right)=2|x|_{\bar{u}}+2\left|\beta_{2}\right|_{\bar{u}}=2|\beta|_{\bar{u}}
$$

To prove that $f$ is injective, assume that $f(\beta)=f\left(\beta^{\prime}\right)$, where $\beta^{\prime}=x^{\prime} \beta_{1}^{\prime} d \beta_{2}^{\prime}$ for some $x^{\prime} \in\{u, \bar{u}\}, \beta_{1}^{\prime} \in \mathcal{D}$, and $\beta_{2}^{\prime} \in \mathcal{B}^{[1]}$. Then

$$
f(\beta)=u \beta_{1} y f\left(\beta_{2}\right)=u \beta_{1}^{\prime} y^{\prime} f\left(\beta_{2}^{\prime}\right)=f\left(\beta^{\prime}\right),
$$

in which $y, y^{\prime} \in\{u, d\}$. Thus $\beta_{1}=\beta_{1}^{\prime}, y=y^{\prime}$, and $f\left(\beta_{2}\right)=f\left(\beta_{2}^{\prime}\right)$. By the induction hypothesis, $f\left(\beta_{2}\right)=f\left(\beta_{2}^{\prime}\right)$ implies that $\beta_{2}=\beta_{2}^{\prime}$, and hence $\beta=\beta^{\prime}$.

To prove that $f$ is surjective, take any $\alpha=u \alpha^{\prime} y \alpha^{\prime \prime}$ in $\mathcal{A}_{n}$, where $y \in\{u, d\}, \alpha^{\prime} \in \mathcal{D}$, and $\alpha^{\prime \prime} \in \mathcal{A}$. By the induction hypothesis, there exists $\beta^{\prime \prime}$ in $\mathcal{B}^{[1]}$ such that $f\left(\beta^{\prime \prime}\right)=\alpha^{\prime \prime}$; so $f\left(u \alpha^{\prime} y \beta^{\prime \prime}\right)=\alpha$. This completes the proof of the first part of the proposition.

Given the first result, the second result may be formulated as saying that the central binomial coefficient $\left(\begin{array}{c}2 n \\ n\end{array}\right)$ is the sum of the ballot numbers $b(n+k, n-k)$ for $k=0,1, \ldots, n$. This is a known fact (see [8, p. 79]). Indeed,

$$
\frac{2 k+1}{n+k+1}\left(\begin{array}{c}
2 n \\
n-k
\end{array}\right)=\left(\begin{array}{c}
2 n \\
n-k
\end{array}\right)-\left(\begin{array}{c}
2 n \\
n-k-1
\end{array}\right),
$$

and hence the sum of these numbers is telescoping.

For a bijective proof of the second part we consider the set of all lattice paths from $(0,0)$ to $(2 n, 0)$. Let $\mathcal{P}_{n}$ be the language over $\{u, d\}$ obtained from these $\left(\begin{array}{c}2 n \\ n\end{array}\right)$ paths via the usual coding, and let $\mathcal{P}=\cup_{n \geq 0} \mathcal{P}_{n}$. The characteristic series of $\mathcal{P}$ satisfies

$$
\mathcal{P}=\epsilon+u \mathcal{D} d \mathcal{P}+d \widehat{\mathcal{D}} u \mathcal{P}
$$


where $\widehat{\mathcal{D}}$ is the image of $\mathcal{D}$ under the involution on $\mathbb{C}\langle\langle u, d\rangle\rangle$ defined by $u \mapsto d$ and $d \mapsto u$; this involution has the effect of reflecting a Dyck path in the $x$-axis. A length preserving bijection $g$ from $\mathcal{B}^{[1]}$ onto $\mathcal{P}$ is then recursively defined by

$$
g(\beta)= \begin{cases}\epsilon & \text { if } \beta=\epsilon, \\ u \beta_{1} d g\left(\beta_{2}\right) & \text { if } \beta=u \beta_{1} d \beta_{2}, \beta_{1} \in \mathcal{D}, \beta_{2} \in \mathcal{B}^{[1]}, \\ d \hat{\beta}_{1} u g\left(\beta_{2}\right) & \text { if } \beta=\bar{u} \beta_{1} d \beta_{2}, \beta_{1} \in \mathcal{D}, \beta_{2} \in \mathcal{B}^{[1]} .\end{cases}
$$

Again, by induction on path-length it follows that $g$ is a bijection.

Example As an illustration of the bijections in the proof of Proposition 3, we have

and
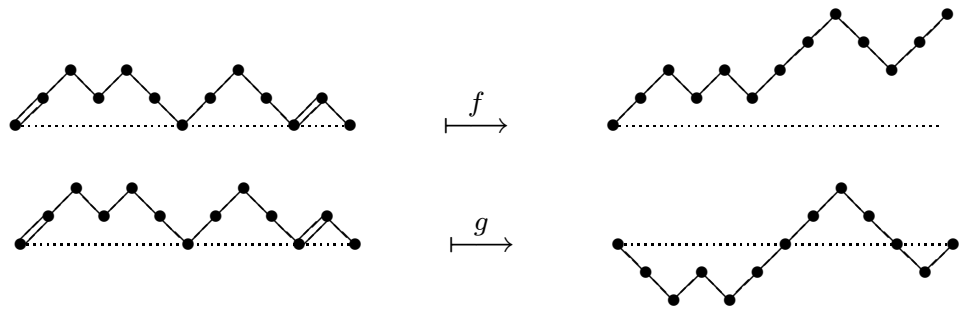

Proposition 4 For $n, k \geq 0$ we have

$$
\left|\mathcal{B}_{n, k}^{[2]}\right|=\sum_{i \geq 0} \frac{2 k+i+1}{n+k+i+1}\left(\begin{array}{c}
k-1 \\
k-i
\end{array}\right)\left(\begin{array}{c}
2 n+i \\
n-k
\end{array}\right) .
$$

Proof From (9) it follows that

$$
B^{[2]}(q, t)=\frac{1-t(1+q) C(t)}{1-t(1+q)(1+C(t))} .
$$

Using (4) we rewrite this as

$$
B^{[2]}(q, t)=\frac{\left(1-q t(C(t))^{2}\right) C(t)}{1-(1+C(t)) q t(C(t))^{2}},
$$

and on expanding the right hand side as a geometric series we get

$$
\left[q^{k}\right] B^{[2]}(q, t)=t^{k} C(t)^{2 k+1}(1+C(t))^{k-1}\left(\delta_{k, 0}+C(t)\right),
$$

where $\delta_{k, 0}$ is 1 if $k=0$, and 0 otherwise. The result is easy to check for $k=0$, so let us assume that $k \geq 1$. Then

$$
\left[q^{k}\right] B^{[2]}(q, t)=t^{k} \sum_{i \geq 0}\left(\begin{array}{c}
k-1 \\
i
\end{array}\right)(C(t))^{3 k-i+1}=t^{k} \sum_{i \geq 0}\left(\begin{array}{c}
k-1 \\
3 k-i
\end{array}\right)(C(t))^{i+1} .
$$

From (7) we get

$$
\begin{aligned}
{\left[t^{n} q^{k}\right] B^{[2]}(q, t) } & =\sum_{i \geq 0} \frac{i+1}{n-k+i+1}\left(\begin{array}{c}
k-1 \\
3 k-i
\end{array}\right)\left(\begin{array}{c}
2 n-2 k+i \\
n-k
\end{array}\right) \\
& =\sum_{i \geq 0} \frac{2 k+i+1}{n+k+i+1}\left(\begin{array}{c}
k-1 \\
i-1
\end{array}\right)\left(\begin{array}{c}
2 n+i \\
n-k
\end{array}\right),
\end{aligned}
$$

which completes the proof. 


\section{$3 \quad$ Segmented permutations}

Let $v=v_{1} v_{2} \cdots v_{n}$ be a word over $\mathbb{N}$ without repeated letters. We define the reduction of $v$, denoted $\operatorname{red}(v)$, by

$$
\operatorname{red}(v)(i)=\left|\left\{j: v_{j} \leq v_{i}\right\}\right| .
$$

In other words, $\operatorname{red}(v)$ is the permutation of $[n]$ obtained from $v$ by replacing the smallest letter in $v$ with 1 , the second smallest with 2 , etc. For instance, $\operatorname{red}(19453)=15342$. We will also need a map that is a kind of inverse to red. For a finite subset $V$ of $\mathbb{N}$, with $n=|V|$, and a permutation $\pi$ of $[n]$, we denote by $\operatorname{red}_{V}^{-1}(\pi)$ the word over $V$ obtained from $\pi$ by replacing $i$ in $\pi$ with the $i$ th smallest element in $V$, for all $i$. Here is an example: If $V=\{1,3,4,5,9\}$ then $\operatorname{red}_{V}^{-1}(15342)=19453$.

Given $\pi$ in $\mathcal{S}_{n}$ and $\sigma$ in $\mathcal{S}_{k}$ ( $\sigma$ is often referred to as a pattern), an occurrence of $\sigma$ in $\pi$ is a subword

$$
o=\pi\left(i_{1}\right) \pi\left(i_{2}\right) \cdots \pi\left(i_{k}\right)
$$

of $\pi$ such that $\operatorname{red}(o)=\sigma$. If, in addition, $i_{r}+1=i_{r+1}$ for each $r=1,2, \ldots, k-1$, then $o$ is a segment-occurrence of $\sigma$ in $\pi$. We say that $\pi$ is $(\sigma)^{k}$-segmented if there are exactly $k$ occurrences of $\sigma$ in $\pi$, each of which is a segment-occurrence of $\sigma$ in $\pi$. A $(\sigma)^{0}$-segmented permutation is usually called $\sigma$-avoiding, and the set of $\sigma$-avoiding permutations of $[n]$ is denoted $\mathcal{S}_{n}(\sigma)$.

If $\pi$ is $(\sigma)^{k}$-segmented for some $k$, then we say that $\pi$ is $\sigma$-segmented. We also define

$$
\mathcal{R}_{n}^{k}(\sigma)=\left\{\pi \in \mathcal{S}_{n}: \pi \text { is }(\sigma)^{k} \text {-segmented }\right\}
$$

and $\mathcal{R}_{n}(\sigma)=\cup_{k \geq 0} \mathcal{R}_{n}^{k}(\sigma)$. In other words, $\mathcal{R}_{n}(\sigma)$ is the set of $\sigma$-segmented permutations of length $n$. Let

$$
R(\sigma ; q, t)=\sum_{k, n \geq 0}\left|\mathcal{R}_{n}^{k}(\sigma)\right| q^{k} t^{n}
$$

The first nontrivial case is $\sigma=12$. A permutation is 12-segmented if all its noninversions are rises. For instance, the permutation 7653412 is 12 -segmented while 7643512 is not (45 is a non-inversion, but not a rise).

Let $\pi \in \mathcal{R}_{n}(12)$ with $n \geq 1$. If the letter 1 precedes the letter $b$ in $\pi$, then $1 b$ is an occurrence of 12 in $\pi$. Thus, either 1 is the last letter in $\pi$, or 1 is the penultimate letter in $\pi$ and 2 is the last letter in $\pi$. In terms of the generating function $R=R(12 ; q, t)$ this amounts to

$$
R=1+t R+q t^{2} R .
$$

So $R$ is a rational function in $t$ and $q$. Extracting coefficients we get

$$
\left|\mathcal{R}_{n}^{k}(12)\right|=\left(\begin{array}{c}
n-k \\
k
\end{array}\right) \text { and }\left|\mathcal{R}_{n}(12)\right|=F_{n},
$$

where $F_{n}$ is the $n$th Fibonacci number (i.e., $F_{n+1}=F_{n}+F_{n-1}$ with $F_{0}=F_{1}=1$ ). This 
is in fact an old result:

$\pi$ is 12 -segmented

$\Longleftrightarrow$ there is no subword $a x b$, with $a<b$ and $x \neq \epsilon$, in $\pi$

$\Longleftrightarrow \pi$ avoids all linear extensions of the poset $\begin{array}{lll}3 & & \\ 1 & & 2\end{array}$

$\Longleftrightarrow \pi$ is $\{123,132,213\}$-avoiding

The $\{123,132,213\}$-avoiding permutations have been enumerated by Simion and Schmidt $[9]$.

In general, to every pattern $\sigma$ there is a set of patterns $\Sigma(\sigma)$ such that a permutation is $\sigma$-segmented precisely when it is $\Sigma(\sigma)$-avoiding. For example,

$$
\Sigma(123)=\{1243,1234,1324,1423,2134,2314\}
$$

these are the linear extensions of the two posets

$\begin{array}{lllll}4 & & & 4 & \\ 1 & & & & \\ 1 & & \\ 2 & & \text { and } & 3 & \\ 1 & & & 1 & \\ 1 & 3 & & 1 & 2\end{array}$

Similarly, we have

$$
\Sigma(132)=\{1243,1342,1423,1432,2143,2413\}
$$

To summarize,

$$
\begin{aligned}
\mathcal{R}_{n}(12) & =\mathcal{S}_{n}(123,132,213) \\
\mathcal{R}_{n}(123) & =\mathcal{S}_{n}(1243,1234,1324,1423,2134,2314) \\
\mathcal{R}_{n}(132) & =\mathcal{S}_{n}(1243,1342,1423,1432,2143,2413) .
\end{aligned}
$$

Theorem 5 Let $k \geq 0$ and $n \geq 3 k$.

The 132-segmented permutations of length $n$ with $k$ occurrences of 132 are in oneto-one correspondence with bicoloured Dyck paths of length $2 n-4 k$ with $k$ red up-steps. Thus

$$
\left|\mathcal{R}_{n}^{k}(132)\right|=\left|\mathcal{B}_{n-2 k, k}\right|=\left(\begin{array}{c}
n-2 k \\
k
\end{array}\right) C_{n-2 k}
$$

where the last equality is a consequence of Proposition 1.

The 123-segmented permutations of length $n$ with $k$ occurrences of 123 are in one-toone correspondence with bicoloured Dyck paths of length $2 n-4 k$ with $k$ red up-steps, each of height less than 2. Thus

$$
\left|\mathcal{R}_{n}^{k}(123)\right|=\left|\mathcal{B}_{n-2 k, k}^{[2]}\right|=\sum_{i \geq 0} \frac{2 k+i+1}{n-k+i+1}\left(\begin{array}{c}
k-1 \\
k-i
\end{array}\right)\left(\begin{array}{c}
2 n-4 k+i \\
n-3 k
\end{array}\right)
$$

where the last equality is a consequence of Proposition 4. 
First proof Let $n$ be a positive integer, and let $\pi$ be a 132-segmented permutation of length $n$. If the letter $n$ is not part of any occurrence of 132, then we can factor $\pi$ as $\pi=\pi_{1} n \pi_{2}$, where $\pi_{1}$ and $\pi_{2}$ are 132-segmented permutations, and $\pi_{2}<\pi_{1}$ (i.e., every letter in $\pi_{2}$ is smaller than every letter in $\pi_{1}$ ). On the other hand, if $n$ is part of an occurrence of 132 , then we can factor $\pi$ as

$$
\pi=\pi_{1} a n b \pi_{2}, \text { where } \pi_{2}<a<b<\pi_{1},
$$

and $\pi_{1}$ and $\pi_{2}$ are 132-segmented permutations. In particular, $a=\left|\pi_{2}\right|+1$ and $b=a+1$. Thus the generating function $R=R(132 ; q, t)$ satisfies the functional equation

$$
R=1+\left(t+q t^{3}\right) R^{2} .
$$

It follows that $R=C\left(t+q t^{3}\right)$, where $C(t)$ is the generating function for the Catalan numbers, and hence $\left[t^{n} q^{k}\right] R=\left|\mathcal{B}_{n-2 k, k}\right|$, as claimed.

Let $\pi \in \mathcal{R}_{n}^{k}(123)$ with $n \geq 1$. Then, either $k=0$ and $\pi$ is 123-avoiding, or $k \geq 1$ and $\pi$ contains at least one occurrence of 123 . Let us focus on the latter case, and let

$$
\pi=\pi_{1} a b c \pi_{2},
$$

where $a b c$ is the leftmost occurrence of 123 in $\pi$. Then $a \pi_{2}$ is $(123)^{k-1}$-segmented and $\pi_{1} c$ is 123 -avoiding, with the additional restriction that $a \pi_{2}$ may not begin with an occurrence of 123. Moreover,

$$
a \pi_{2}<b<\pi_{1} c,
$$

or else a non segment-occurrence of 123 would be present. With regard to the generating function $R=R(123 ; q, t)$ this decomposition of 123 -segmented permutations amounts to the functional equation

$$
R=C+q t(C-1)(\tilde{R}-1),
$$

where $C=C(t)$ is the generating function of the Catalan numbers, and the coefficient of $q^{k} t^{n}$ in $\tilde{R}=\tilde{R}(q, t)$ is the number of $(123)^{k}$-segmented permutations of length $n$ that do not begin with an occurrence of 123 . Considering the decomposition above in the special case when $\pi_{1}$ is the empty word, we see that $t^{2} q(\tilde{R}-1)$ is the generating function of the number of 123 -segmented permutations that begin with an occurrence of 123 ; so

$$
R=\tilde{R}+q t^{2}(\tilde{R}-1) .
$$

Solving equations (14) and (15) for $R$, eliminating $\tilde{R}$, we get

$$
R=\frac{\left(1-q t^{3} C^{2}\right) C}{1-(1+C) q t^{3} C^{2}} .
$$

It follows from (11) that $R=B^{[2]}\left(q t^{2}, t\right)$, as claimed. 
Second proof We shall define a bijection

$$
f: \mathcal{R}(132) \rightarrow \mathcal{B}^{[2]},
$$

such that $|f(\pi)|=2(n-2 k)$ and $|f(\pi)|_{\bar{u}}=k$ whenever $\pi \in \mathcal{R}_{n}^{k}(132)$. Our definition of $f$ will be recursive and we start by defining that $f(\epsilon)=\epsilon$. Now, assume that $n$ is a positive integer, and let $\pi$ be a 132-segmented permutation of length $n$. As in the first proof, if the letter $n$ is not part of any occurrence of 132 , then we can factor $\pi$ as $\pi=\pi_{1} n \pi_{2}$, where $\pi_{1}$ and $\pi_{2}$ are 132-segmented permutations, and $\pi_{2}<\pi_{1}$; in this case we define

$$
f(\pi)=u(f \circ \text { red })\left(\pi_{1}\right) d(f \circ \text { red })\left(\pi_{2}\right) .
$$

If $n$ is part of an occurrence of 132 , then we can factor $\pi$ as $\pi=\pi_{1} a n b \pi_{2}$ where $\pi_{2}<a<$ $b<\pi_{1}$ and $\pi_{1}$ and $\pi_{2}$ are 132-segmented permutations; in this case we define

$$
f(\pi)=\bar{u}(f \circ \operatorname{red})\left(\pi_{1}\right) d(f \circ \text { red })\left(\pi_{2}\right) .
$$

For any $\beta$ in $\mathcal{B}$, let

$$
\lambda(\beta)=\frac{1}{2}|\beta|+2|\beta|_{\bar{u}}=|\beta|_{u}+3|\beta|_{\bar{u}} .
$$

Using induction, it is plain to show that the inverse of $f$ is given by

$$
\begin{aligned}
f^{-1}(\epsilon) & =\epsilon ; \\
f^{-1}\left(u \beta_{1} d \beta_{2}\right) & =\left(\operatorname{red}_{V_{1}}^{-1} \circ f^{-1}\right)\left(\beta_{1}\right) n\left(\operatorname{red}_{V_{2}}^{-1} \circ f^{-1}\right)\left(\beta_{2}\right),
\end{aligned}
$$

where $n=\lambda\left(\beta_{1}\right)+\lambda\left(\beta_{2}\right)+1, V_{1}=\left[\lambda\left(\beta_{2}\right)+1, n-1\right]$, and $V_{2}=\left[1, \lambda\left(\beta_{2}\right)\right]$;

$$
f^{-1}\left(\bar{u} \beta_{1} d \beta_{2}\right)=\left(\operatorname{red}_{V_{1}}^{-1} \circ f^{-1}\right)\left(\beta_{1}\right) \operatorname{anb}\left(\operatorname{red}_{V_{2}}^{-1} \circ f^{-1}\right)\left(\beta_{2}\right),
$$

where $a=\lambda\left(\beta_{2}\right)+1, b=a+1, n=\lambda\left(\beta_{1}\right)+b+1, V_{1}=[b+1, n-1]$, and $V_{2}=[1, a-1]$.

To find a bijective proof of the second part of Theorem 5 we will first discuss a decomposition of paths in $\mathcal{B}^{[2]}$ which is similar to the decomposition of permutations in $\mathcal{R}(123)$ underlying (14). Let $\beta \in \mathcal{B}^{[2]}$. If there is a leftmost occurrence of $\bar{u}$ in $\beta$ then the height of that $\bar{u}$ must be either 0 or 1 . Thus we have

$$
\mathcal{B}^{[2]}=\mathcal{D}+\mathcal{D} \bar{u} \mathcal{B}^{[1]} d \mathcal{B}^{[2]}+\mathcal{D} u \mathcal{D} \bar{u} \mathcal{D} d \mathcal{B}^{[1]} d \mathcal{B}^{[2]}
$$

whose commutative counterpart is

$$
\begin{aligned}
B^{[2]} & =C+q t C B^{[1]} B^{[2]}+q t^{2} C^{3} B^{[1]} B^{[2]} \\
& =C+q t^{-1}\left(t C+t^{2} C^{3}\right) t B^{[1]} B^{[2]} .
\end{aligned}
$$

Since $C=1+t C^{2}$, the factor $t C+t^{2} C^{3}$ simplifies to $C-1$. Moreover, if we let $\tilde{\mathcal{B}}^{[2]}$ denote the set of paths in $\mathcal{B}^{[2]}$ whose first step is $u$ (i.e., not $\bar{u}$ ), then

$$
\tilde{\mathcal{B}}^{[2]}=\epsilon+u \mathcal{B}^{[1]} d \mathcal{B}^{[2]},
$$



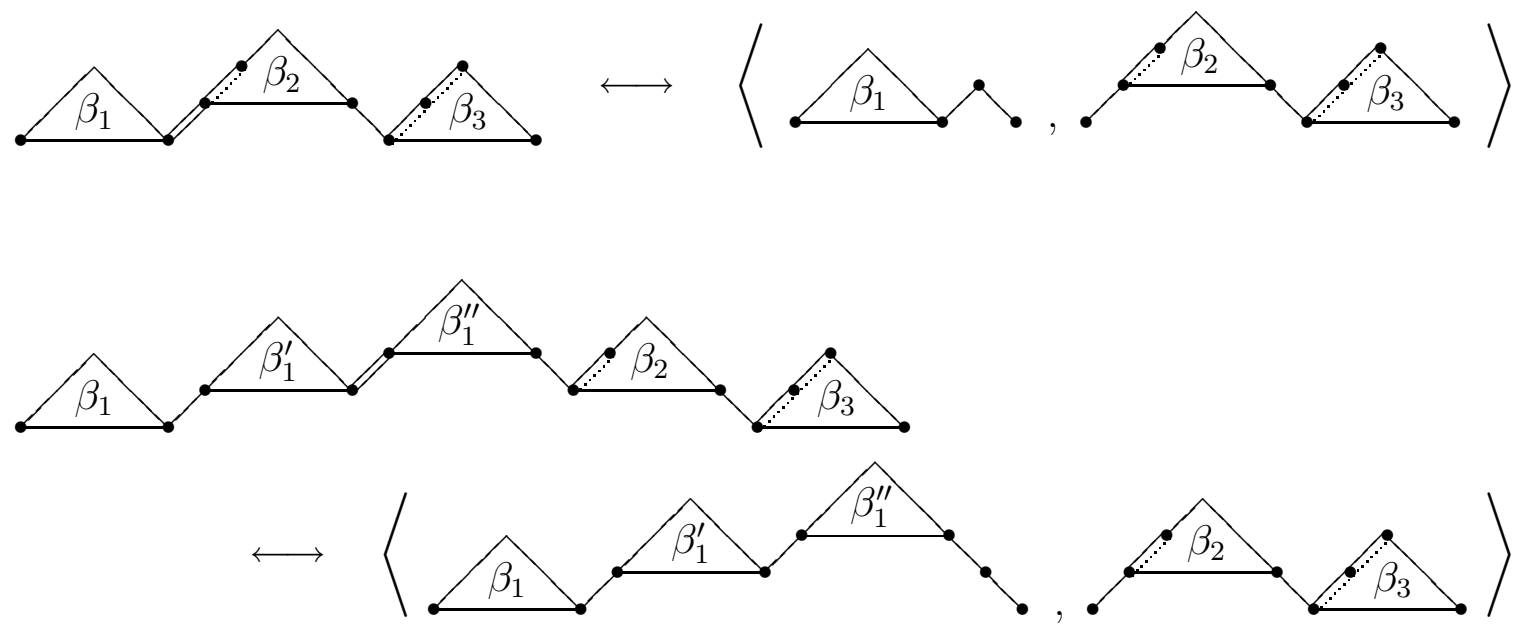

Figure 1: The bijection $\mathcal{B}^{[2]} \backslash \mathcal{D} \stackrel{\Phi}{\cong}(\mathcal{D} \backslash\{\epsilon\}) \times\left(\tilde{\mathcal{B}}^{[2]} \backslash\{\epsilon\}\right)$.

and, as a consequence, $t B^{[1]} B^{[2]}=\tilde{B}^{[2]}-1$. Thus (18) can be rewritten as

$$
B^{[2]}=C+q t^{-1}(C-1)\left(\tilde{B}^{[2]}-1\right),
$$

which should be compared to (14). This suggests that we should be able to uniquely decompose any path $\beta$ in $\mathcal{B}^{[2]} \backslash \mathcal{D}$ into two nonempty paths $\beta^{\prime} \in \mathcal{D}$ and $\beta^{\prime \prime} \in \tilde{B}^{[2]}$ such that $|\beta|=\left|\beta^{\prime}\right|+\left|\beta^{\prime \prime}\right|-1$ and $|\beta|_{\bar{u}}=\left|\beta^{\prime \prime}\right|_{\bar{u}}+1$. Indeed, using (17), (19) and

$$
\mathcal{D}=\epsilon+\mathcal{D} u d+\mathcal{D} u \mathcal{D} u \mathcal{D} d d
$$

such a decomposition is defined by the map

$$
\begin{aligned}
\beta_{1} \bar{u} \beta_{2} d \beta_{3} & \mapsto\left\langle\beta_{1} u d, u \beta_{2} d \beta_{3}\right\rangle, \\
\beta_{1} u \beta_{1}^{\prime} \bar{u} \beta_{1}^{\prime \prime} d \beta_{2} d \beta_{3} & \mapsto\left\langle\beta_{1} u \beta_{1}^{\prime} u \beta_{1}^{\prime \prime} d d, u \beta_{2} d \beta_{3}\right\rangle,
\end{aligned}
$$

where $\beta_{1}, \beta_{1}^{\prime}, \beta_{1}^{\prime \prime} \in \mathcal{D}, \beta_{2} \in \mathcal{B}^{[1]}$, and $\beta_{3} \in \mathcal{B}^{[2]}$. We denote by $\Phi$ the inverse of this map; it is obtained by simply reversing the arrows. See Figure 3 for a schematic diagram of $\Phi$.

Let $h$ be any bijection from $\mathcal{S}_{n}(123)$ to $\mathcal{D}_{n}$. For definiteness, we can take $h$ to be the bijection $\Psi$ given by Krattenthaler in [5, p. 522]. (A description of $\Psi$ can be found in the example following this proof.) We shall define a bijection

$$
g: \mathcal{R}(123) \rightarrow \mathcal{B}^{[2]}
$$

such that $|g(\pi)|=2(n-2 k)$ and $|g(\pi)|_{\bar{u}}=k$, whenever $\pi \in \mathcal{R}_{n}^{k}(123)$. If $\pi$ avoids 123 then let $g(\pi)=h(\pi)$. If $\pi$ does not avoid 123 then, as in the first proof, we can write $\pi=\pi_{1} a b c \pi_{2}$, where $a b c$ is the leftmost occurrence of 123 in $\pi$; in this case, we let

$$
g(\pi)=\Phi\left\langle(g \circ \operatorname{red})\left(\pi_{1} c\right),(g \circ \operatorname{red})\left(a \pi_{2}\right)\right\rangle .
$$

Proving that $g$ is invertible is similar to proving that $f$ is invertible. 
We remark that the bijection $f$ from the first part of the preceding proof maps 132avoiding permutations onto Dyck paths. In fact, the restriction of $f$ to $\mathcal{S}(132)$ is a bijection due to Krattenthaler [5, p. 512].

Example The permutation 846572931 is 132 -segmented. It has two occurrences of 132 , namely 465 and 293. We illustrate the bijection $f$, from the first part of the preceding proof, by finding the image of 846572931 under $f$ :

$$
\begin{gathered}
f(846572931)=\bar{u} f(84657) d f(1)=\bar{u} u d f(4657) d u d= \\
=\bar{u} u d u f(465) d d u d=\bar{u} u d u \bar{u} d d d u d .
\end{gathered}
$$

For convenience we have not reduced the permutations in the intermediate steps.

To give an example of how $g$, from the second part of the preceding proof, is applied, we first need to describe Krattenthaler's [5, p. 522] bijection $\Psi$ from $\mathcal{S}_{n}(123)$ to $\mathcal{D}_{n}$. Let $\pi=a_{1} a_{2} \cdots a_{n}$ be a 123-avoiding permutation. A right-to-left maximum is an element $a_{i}$ such that $a_{i}>a_{j}$ for all $j>i$. Let the right-to-left maxima in $\pi$ be $m_{1}, m_{2}, \ldots, m_{s}$, from right to left, so that

$$
\pi=\pi_{s} m_{s} \cdots \pi_{2} m_{2} \pi_{1} m_{1},
$$

where $\pi_{i}$ is the subword of $\pi$ between $m_{i+1}$ and $m_{i}$. If there is an occurrence $a b$ of 12 in $\pi_{i}$ then $a b m_{i}$ is an occurrence of 123 in $\pi$. Therefore, the elements in $\pi_{i}$ are in decreasing order. Moreover, we have $\pi_{i}<\pi_{i+1}$.

The Dyck path $\Psi(\pi)$ is generated from right to left: Read $\pi$ from right to left. Any right-to-left maximum $m_{i}$ is translated into $m_{i}-m_{i-1}$ up-steps (with the convention $\left.m_{0}=0\right)$. Any subword $\pi_{i}$ is translated into $\left|\pi_{i}\right|+1$ down-steps.

We are now ready for an illustration of the bijection $g$. The permutation 957841362 is 123-segmented. It has two occurrences of 123 , namely 578 and 136 . To find the image of 957841362 under $g$ we proceed as follows:

$$
\begin{aligned}
g(957841362) & =\Phi\langle(g \circ \text { red })(98),(g \circ \text { red })(541362)\rangle, \\
(g \circ \text { red })(98) & =\Psi(21)=u d u d, \\
(g \circ \text { red })(541362) & =\Phi\langle(g \circ \text { red })(546),(g \circ \text { red })(12)\rangle, \\
(g \circ \text { red })(546) & =\Psi(213)=u u u d d d, \\
(g \circ \text { red })(12) & =\Psi(12)=u u d d, \\
\Phi\langle u u u d d d, u u d d\rangle & =u \bar{u} u d d u d d, \\
\Phi\langle u d u d, u \bar{u} u d d u d d\rangle & =u d \bar{u} \bar{u} u d d u d d .
\end{aligned}
$$

Thus $g(957841362)=u d \bar{u} \bar{u} u d d u d d$.

Corollary 6 For $k \geq 0$ and $n \geq 0$ we have

$$
\left|R_{n}^{k}(123)\right| \leq\left|\mathcal{R}_{n}^{k}(132)\right|
$$

Proof The result follows immediately from $\mathcal{B}_{n, k}^{[2]} \subseteq \mathcal{B}_{n, k}$ and Theorem 5 . 
Corollary 7 The generating functions $R(132 ; q, t)$ and $R(123 ; q, t)$ admit the following continued fraction expansions:

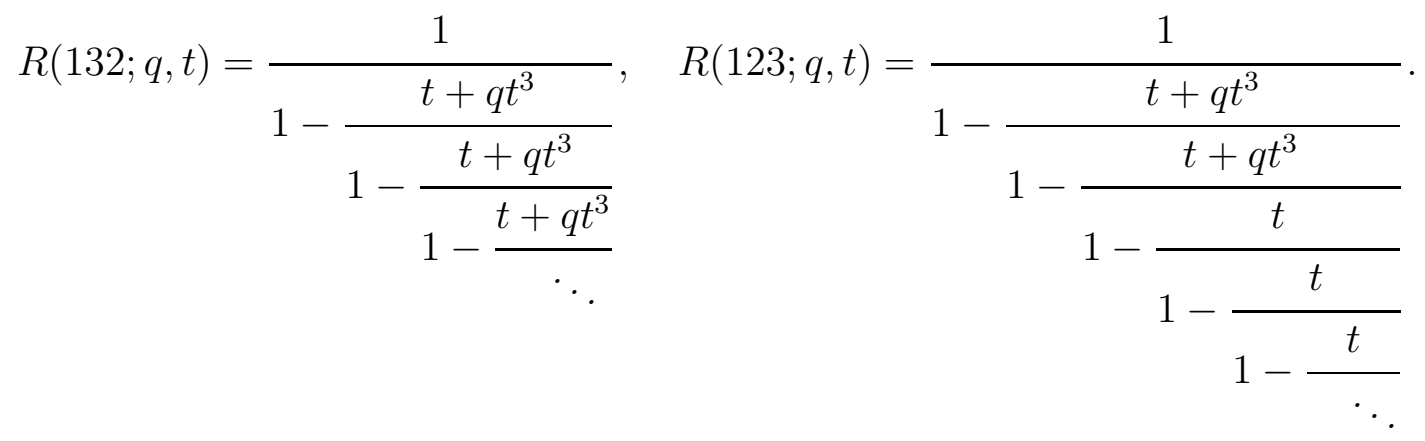

Proof The first identity to prove is simply iterating the formula (13). The second identity follows from $R=B^{[2]}\left(q t^{2}, t\right),(9)$, and iteration of $C(t)=1 /(1-t C(t))$.

Proposition 8 The generating function

$$
R(123,132 ; p, q, t)=\sum_{\pi \in \mathcal{R}(123) \cap \mathcal{R}(132)} p^{(123) \pi} q^{(132) \pi} t^{|\pi|}
$$

counting $\{123,132\}$-segmented permutations by occurrences of 123 and 132 is the following rational function:

$$
R(123,132 ; p, q, t)=\frac{1-t}{1-2 t-(p+q) t^{3}}=\frac{1}{1-\frac{t+(p+q) t^{3}}{1-t}} .
$$

First proof Let $n$ be a positive integer, and let $\pi$ be a $\{123,132\}$-segmented permutation of length $n$. We distinguish between three cases:

(a) If the letter $n$ is not part of any occurrence of 123 or 132, then we can factor $\pi$ as $\pi=\pi_{1} n \pi_{2}$, where $\pi_{1}$ is 12-avoiding, $\pi_{2}$ is $\{123,132\}$-segmented, and $\pi_{2}<\pi_{1}$.

(b) If the letter $n$ is part of an occurrence of 123, then we can factor $\pi$ as $\pi=\pi_{1} a b n \pi_{2}$, where $\pi_{1}$ is 12-avoiding, $\pi_{2}$ is $\{123,132\}$-segmented, and $\pi_{2}<a<b<\pi_{1}$.

(c) If the letter $n$ is part of an occurrence of 132 , then we can factor $\pi$ as $\pi=\pi_{1} a n b \pi_{2}$, where $\pi_{1}$ is 12-avoiding, $\pi_{2}$ is $\{123,132\}$-segmented, and $\pi_{2}<a<b<\pi_{1}$.

It is clear that an occurrence of 123 can not overlap with an occurrence of 132 without creating a non-segment occurrence of 123 or 132 . Therefore, the cases (b) and (c) are mutually exclusive. Thus the generating function $R=R(123,132 ; p, q, t)$ satisfies

$$
R=1+R(12 ; 0, t)\left(t+p t^{3}+q t^{3}\right) R,
$$

where $R(12 ; 0, t)=1 /(1-t)$ is the generating function for 12 -avoiding permutations. Solving (20) for $R$ we obtain the desired result. 
Second proof To give a bijective proof of Proposition 8 we consider lattice paths with three different types of up-steps: let $\mathcal{T}$ be the language over $\{u, \bar{u}, \overline{\bar{u}}, d\}$ whose characteristic series is implicitly given by

$$
\mathcal{T}=\epsilon+(u+\bar{u}+\overline{\bar{u}})(u d)^{*} d \mathcal{T}
$$

where $(u d)^{*}=\epsilon+u d+u d u d+\cdots$. We may think of a word in the language $\mathcal{T}$ as a 3 -coloured Dyck path whose $u$-steps are of height 0 or 1 , and whose $\bar{u}$ - and $\overline{\bar{u}}$-steps are of height 0 . Applying the substitution $\mu: \mathbb{C}\langle\langle u, \bar{u}, \overline{\bar{u}}, d\rangle\rangle \rightarrow \mathbb{C}[[p, q, t]]$ defined by

$$
\mu=\{u \mapsto 1, \bar{u} \mapsto p, \overline{\bar{u}} \mapsto q, d \mapsto t\}
$$

we see that $R(123,132, p, q, t)=\mu(\mathcal{T})\left(p t^{2}, q t^{2}, t\right)$. We shall give a bijection

$$
f: \mathcal{R}(123) \cap \mathcal{R}(132) \rightarrow \mathcal{T}
$$

such that $|\pi|=|\beta|_{u}+3\left(|\beta|_{\bar{u}}+|\beta|_{\bar{u}}\right)$, where $\beta=f(\pi)$. Following the decomposition given in the first proof, we recursively define $f$ as follows:

$$
\begin{aligned}
f(\epsilon) & =\epsilon, \\
f\left(\pi_{1} n \pi_{2}\right) & =u(f \circ \mathrm{red})\left(\pi_{1}\right) d(f \circ \mathrm{red})\left(\pi_{2}\right), \\
f\left(\pi_{1} a b n \pi_{2}\right) & =\bar{u}(f \circ \mathrm{red})\left(\pi_{1}\right) d(f \circ \mathrm{red})\left(\pi_{2}\right), \\
f\left(\pi_{1} a n b \pi_{2}\right) & =\overline{\bar{u}}(f \circ \text { red })\left(\pi_{1}\right) d(f \circ \mathrm{red})\left(\pi_{2}\right) .
\end{aligned}
$$

It is straightforward, but tedious, to give the inverse of $f$.

Example The permutation 875963124 is $\{123,132\}$-segmented. It has one occurrence of each of the patterns 123 and 132, namely 124 and 596. To illustrate the second proof of Proposition 8 we find the image of 875963124 under $f$ :

$$
f(875963124)=\overline{\bar{u}} f(87) d f(3124)=\overline{\bar{u}} u d f(7) d \bar{u} f(3) d=\overline{\bar{u}} u d u d d \bar{u} u d d .
$$

\section{Acknowledgments}

I thank Petter Brändén for several discussions on the subject of this paper. I also thank Einar Steingrímsson for proofreading a preliminary version of this article. 


\section{Tables}

\begin{tabular}{|c|c|c|c|c|c|c|c|c|}
\hline$\overline{n \backslash k}$ & 0 & 1 & 2 & 3 & 4 & 5 & 6 & 7 \\
\hline 0 & 1 & & & & & & & \\
\hline 1 & 1 & 1 & & & & & & \\
\hline 2 & 2 & 4 & 2 & & & & & \\
\hline 3 & 5 & 15 & 15 & 5 & & & & \\
\hline 4 & 14 & 56 & 84 & 56 & 14 & & & \\
\hline 5 & 42 & 210 & 420 & 420 & 210 & 42 & & \\
\hline 6 & 132 & 792 & 1980 & 2640 & 1980 & 792 & 132 & \\
\hline 7 & 429 & 3003 & 9009 & 15015 & 15015 & 9009 & 3003 & 429 \\
\hline
\end{tabular}

$\left|\mathcal{B}_{n, k}^{[1]}\right|:$

\begin{tabular}{crrrrrrrr}
\hline$n \backslash k$ & 0 & 1 & 2 & 3 & 4 & 5 & 6 & 7 \\
\hline 0 & 1 & & & & & & & \\
1 & 1 & 1 & & & & & & \\
2 & 2 & 3 & 1 & & & & & \\
3 & 5 & 9 & 5 & 1 & & & & \\
4 & 14 & 28 & 20 & 7 & 1 & & & \\
5 & 42 & 90 & 75 & 35 & 9 & 1 & & \\
6 & 132 & 297 & 275 & 154 & 54 & 11 & 1 & \\
7 & 429 & 1001 & 1001 & 637 & 273 & 77 & 13 & 1 \\
\hline
\end{tabular}

$\left|\mathcal{B}_{n, k}^{[2]}\right|:$

\begin{tabular}{crrrrrrrr}
\hline$n \backslash k$ & 0 & 1 & 2 & 3 & 4 & 5 & 6 & 7 \\
\hline 0 & 1 & & & & & & & \\
1 & 1 & 1 & & & & & & \\
2 & 2 & 4 & 2 & & & & & \\
3 & 5 & 14 & 13 & 4 & & & & \\
4 & 14 & 48 & 62 & 36 & 8 & & & \\
5 & 42 & 165 & 264 & 217 & 92 & 16 & & \\
6 & 132 & 572 & 1066 & 1104 & 670 & 224 & 32 & \\
7 & 429 & 2002 & 4186 & 5130 & 3965 & 1912 & 528 & 64 \\
\hline
\end{tabular}




\section{References}

[1] T. Chow and J. West. Forbidden subsequences and Chebyshev polynomials. Discrete Math., 204(1-3), 119-128, 1999.

[2] S. Elizalde and M. Noy. Enumeration of subwords in permutations. In Formal power series and algebraic combinatorics (Tempe, 2001), 179-189, Arizona State University, 2001.

[3] S. Kitaev. Multi-avoidance of generalised patterns. Discrete Math., 260(1-3), 89-100, 2003.

[4] S. Kitaev and T. Mansour. Simultaneous avoidance of generalized patterns. Ars Combinatoria, Vol. LXXV, 2005.

[5] C. Krattenthaler. Permutations with restricted patterns and Dyck paths. Advances in Applied Math., 27:510, 510-530, 2001.

[6] G. Kreweras, Sur les éventails de segments, Cahiers du BURO 15, 3-41, 1970.

[7] T. Mansour and A. Vainshtein. Counting occurrences of 132 in a permutation. Advances in Applied Math., 28:2, 329 - 344, 2002.

[8] J. Riordan. Combinatorial identities. John Wiley \& Sons Inc., New York, 1968.

[9] R. Simion and F. W. Schmidt. Restricted permutations. European J. Combin., 6(4), 383-406, 1985. 